\title{
Population genomics reveals additive and replacing horizontal gene transfers in the emerging pathogen Dickeya solani
}

\author{
Slimane Khayi ${ }^{1,2}$, Pauline Blin ${ }^{1}$, Jacques Pédron ${ }^{3}$, Teik-Min Chong ${ }^{4}$, Kok-Gan Chan $^{4}$, Mohieddine Moumni ${ }^{2}$, \\ Valérie Hélias ${ }^{5,6}$, Frédérique Van Gijsegem ${ }^{7}$ and Denis Faure ${ }^{1 *}$
}

\begin{abstract}
Background: Dickeya solani is an emerging pathogen that causes soft rot and blackleg diseases in several crops including Solanum tuberosum, but little is known about its genomic diversity and evolution.

Results: We combined Illumina and PacBio technologies to complete the genome sequence of D. solani strain 3337 that was used as a reference to compare with 19 other genomes (including that of the type strain $1 \mathrm{PO} 2222^{\top}$ ) which were generated by Illumina technology. This population genomic analysis highlighted an unexpected variability among $D$. solani isolates since it led to the characterization of two distinct sub-groups within the $D$. solani species. This approach also revealed different types of variations such as scattered SNP/InDel variations as well as replacing and additive horizontal gene transfers (HGT). Infra-species (between the two D. solani sub-groups) and inter-species (between D. solani and D. dianthicola) replacing HGTs were observed. Finally, this work pointed that genetic and functional variation in the motility trait could contribute to aggressiveness variability in D. solani.
\end{abstract}

Conclusions: This work revealed that $D$. solani genomic variability may be caused by SNPS/InDels as well as replacing and additive HGT events, including plasmid acquisition; hence the $D$. solani genomes are more dynamic than that were previously proposed. This work alerts on precautions in molecular diagnosis of this emerging pathogen.

Keywords: Dickeya, Soft rot, Potato, Population genomics, Horizontal gene transfer

\section{Background}

Pectinolytic bacteria belonging to the Dickeya and Pectobacterium genera are pathogens that cause soft rot and blackleg diseases in a wide range of plants and crops including Solanum tuberosum [1,2]. These phytopathogens produce plant cell-wall degrading enzymes that are able to macerate the tuber and stem tissues, thus provoking the disease symptoms [3]. Since 2000s, the emerging D. solani species has been proposed as a contributor to the increased incidence of blackleg and soft rot diseases on potato crop in Europe and the Mediterranean basin [4]. The D. solani species has been officially described recently [5].

Little is known about the ecological and genetic traits that may support the relative success of $D$. solani in

\footnotetext{
* Correspondence: denis.faure@i2bc.paris-saclay.fr

${ }^{1}$ Institute for Integrative Biology of the Cell (I2BC), CNRS CEA Univ. Paris-Sud, Université Paris-Saclay, Saclay Plant Sciences, Avenue de la Terrasse, 91198 Gif-sur-Yvette cedex, France

Full list of author information is available at the end of the article
}

invading potato fields [6, 7]. D. solani can initiate disease from a low inoculum level in warm climates and was described in some studies to spread more easily through vascular tissues than other Dickeya species $[4,8]$. Besides classical intergenic spacers 16S-23S rDNA [9], several molecular studies have proposed different marker genes for the identification of $D$. solani strains collected from potato and ornamental plants, such as dnaX [10], $r e c A$ [11] and $f l i C$ [12]. At whole genome level, genomic and metabolic comparisons of two D. solani strains Ds0432-1 (isolated in Finland) and 3337 (isolated in France) vs. $D$. dadantii 3937 indicated a conserved synteny between the two species, but also the presence of distinctive traits $[13,14]$. D. solani and $D$. dadantii diverged in their battery of non-ribosomal peptide/polyketide synthase clusters, T5SS/T6SS-related toxin-antitoxin systems and several metabolic abilities. Some of these traits would contribute to the successful invasion of this pathogen $[13,14]$. 
More recently, a reverse genetic approach revealed that the virulence master regulators are quite the same in $D$. solani and D. dadantii [7].

The analysis of population genome structure and dynamics, including additive or replacing horizontal gene transfer (HGT) may bring valuable clues on the mechanisms of emergence of $D$. solani. While additive HGT allows the acquisition of novel genes by a population [15-20], replacing HGT provokes the replacement of an allele by another from close relatives [21]. HGT events inform about the genome diversification and adaptation processes, but also on the companion populations that the pathogens met during the emergence and dissemination steps. Replacing HGT is also of a major stake in pathogen diagnostic, as it may provoke false identification when the alleles exchanged by replacing HGT are used as molecular taxonomic markers.

Here, we analyzed the whole genome polymorphism of 20 D. solani isolates, including the type strain $\mathrm{IPO} 2222^{\mathrm{T}}$, collected from different geographic locations, dates of isolation and plant hosts. We combined Illumina and PacBio technologies to complete the $3337 \mathrm{D}$. solani strain genome that we used as a reference in the comparative genomics. While most strains belonged to a core-population that exhibited less than one hundred variant positions between two given genomes, some other genomes revealed massive replacing HGT from the companion pathogen $D$. dianthicola and a plasmid acquisition from Burkholderia ambifaria. Moreover, we were able to correlate SNPs in virulence genes with a decrease in aggressiveness, highlighting the power of genomics as a tool to reveal functional variability in $D$. solani population. To our knowledge this is the first study that reports whole genome analysis of a D. solani population and describes its diversity.

\section{Results}

Complete genome of the D. solani 3337

The D. solani 3337 genome was previously sequenced by Illumina technology using two libraries (mate-pair and paired-end) and de novo assembled in a high quality draft genome deposited at NCBI [22]. In this work, the 3337 D. solani genome was re-sequenced using PacBio technology. The PacBio sequencing generated six contigs (2 47362 pb, 1512701 bp, 894591 bp, 49337 bp, $10627 \mathrm{bp}$, and $4290 \mathrm{bp}$ ) with an average 150 fold coverage. The published Illumina-scaffolding was confirmed and the remaining gaps were filled using the PacBio contigs. Hence, combining the Illumina and PacBio sets of sequences, we obtained a complete sequence of the unique circular chromosome (4 922460 bp). The RAST annotation generated 4.536 CDS and 97 RNAs. The D. solani 3337 complete genome was used as a reference for comparative genomics.

\section{Positioning the sequenced $\mathrm{D}$. solani strains within the Dickeya genus}

In addition to D. solani 3337, 19 D. solani strains including the type strain IPO $2222^{\mathrm{T}}$ were collected at different years and geographical locations (Additional file 1: Table S1) and their genomes sequenced by Illumina technology. All these draft and complete genomes were used in multi-locus sequence analysis (MLSA) and average nucleotide identity (ANI) calculation. For MLSA, the eleven concatenated rpoD, gyrB, recA, rpoS, dnaX, dnaA, gapA, fusA, rplB, purA, gyrA housekeeping genes (17 $298 \mathrm{bp}$ ) were aligned to construct a relation-tree using Neighbor-Joining method, the evolutionary distances were computed using the Maximum Composite Likelihood method [23]. All the D. solani (Dsl) isolates were grouped in a same cluster that was separated from the other pectinolytic enterobacteria (Fig. 1). Noticeably, the strain Dsl 0512 was the unique strain that was consistently distant from the other D. solani strains. As previously reported [5], within the genus Dickeya the most related species to $D$. solani were $D$. dadantii and $D$. dianthicola. ANI values which were calculated using the strain Dsl 3337 as a reference were in accordance with the MLSA clustering. All the $D$. solani strains exhibited an ANI value

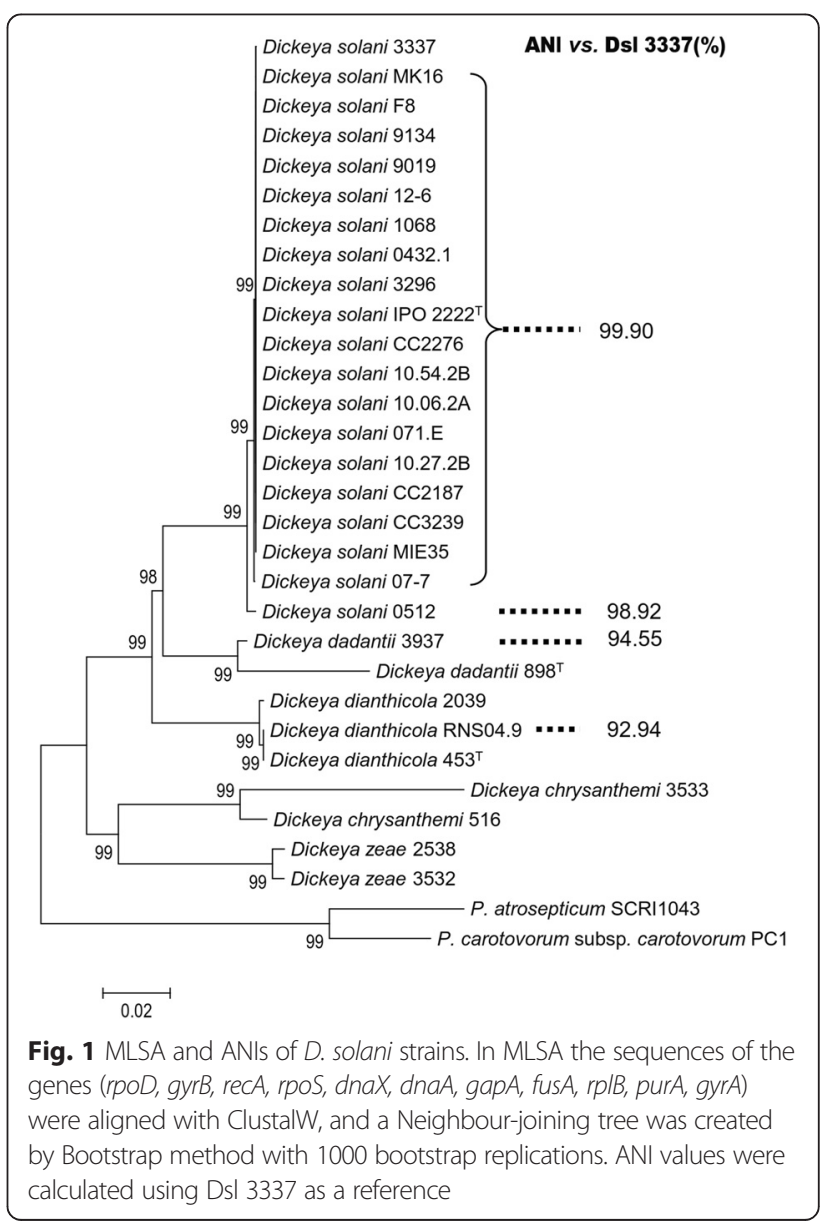


equal to or above $99.9 \%$, but that of Dsl 0512 was below $99 \%$. Among strains of the closest species, D. dadantii and $D$. dianthicola ANI values dropped to $94 \%$ and $92 \%$, respectively.

\section{Overview of the SNP and InDel variations in D. solani genomes}

Illumina reads of the $D$. solani strains were mapped on the complete genome sequence of Dsl 3337. The percentage of mapped reads was above $99 \%$ for all strains with the exception of Dsl 9019 (98.08 \%) and 0512 (92.34 \%) (Additional file 1: Table S2). The mapping vs. Dsl 3337, which reached a high mean coverage value (between 400 and 900), allowed us to identify variations (SNPs and InDels) in each of the genomes (Additional file 1: Table S3). According to the number of variations, the $D$. solani strains could be clustered into three groups. The first group, which we thereafter term as the core-population, encompassed most of the strains (including IPO $2222^{\mathrm{T}}$ and the reference Dsl 3337) with a variation number ranging from 43 to 85 . In the second group were the strains Dsl 07-7, 9019 and 9134 with a variation number between 1454 and 3433. The third group consisted in the only strain Dsl 0512 with a very high variation number that reached 37493 . RAST annotation of the strain Dsl 3337 was used to position the variations in or out coding DNA sequences (CDSs), as well as to identify non-synonymous variations in CDSs (Additional file 1: Table S3). Non-synonymous variations ranged between 14 and $21 \%$ of the total number of variations, hence only 6 to 18 non-synonymous variations were identified in strains of the $D$. solani corepopulation (Additional file 1: Table S3).

\section{Heterogeneous distribution of the SNP and InDel variations} in D. solani genes

We refined our analysis by calculating the number of genes (CDSs) that were affected by SNPs and InDels as well as non-synonymous variations (Fig. 2a-b). In the corepopulation, 9 to 17 genes exhibited variations and about one half of them (4 to 10) harbored non-synonymous variations. In Dsl 07-7, 9019 and 9134, 56 to 144 genes were affected; and among them, 46 to 81 contained nonsynonymous variations. In Dsl 0512, 2760 genes, hence half of the genome showed variations. To compare variation abundance in genes, a mean value of the number of all variations (synonymous and non-synonymous) per affected gene was calculated (Fig. 2c). In the core-population, this value ranged from 2 to 5. In Dsl 0512 and 07-7, it was similar (11 and 9, respectively) while the highest value was observed in Dsl 9134 and 9019 (45 and 42, respectively). Overall, these analyses revealed that Dsl 0512, 07-7, 9134 and 9019 harbored genes with different numbers of variations as compared to those in the

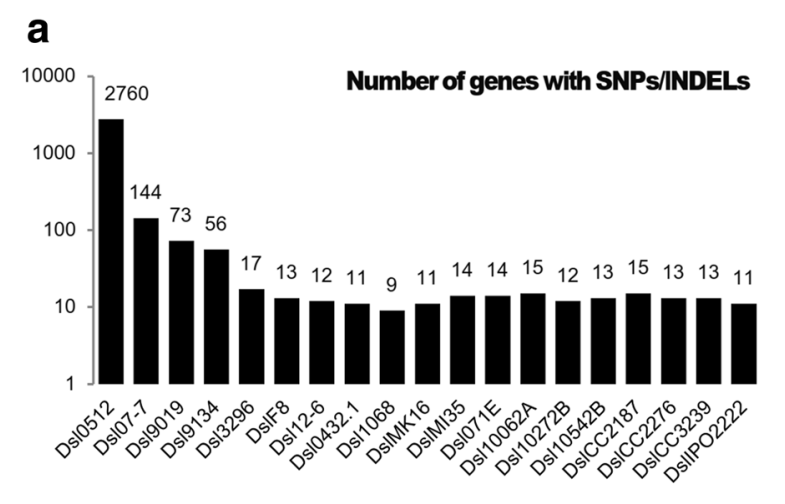

b

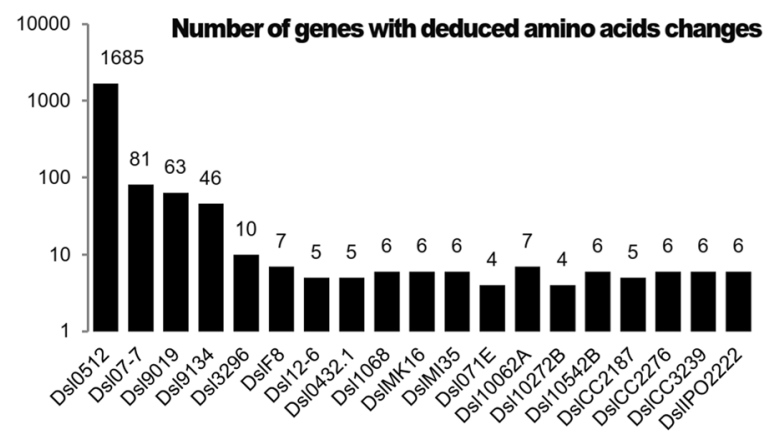

C

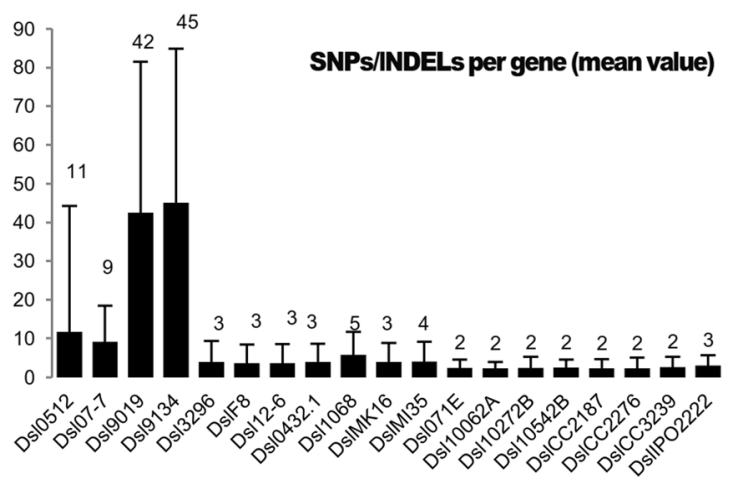

Fig. 2 Number of genes affected by variations (SNPs and InDels). a Total number of genes affected by the variations for each strain. b Number of genes revealing amino acids change further variation. c The average number of variations affecting the genes in each strain

core-population, suggesting putative HGTs from distinct sources.

Finally, all these different variations were positioned along the Dsl chromosome (Fig. 3). In the corepopulation, the rare variations appeared to be scattered with a mean distribution of 0.015 variations per $\mathrm{kbp}$ (when all SNPs and InDels were counted) and 0.012 variations per gene (when only SNPs and InDels in CDSs were counted). In Dsl 9134, 9019 and 07-7, most of the variations affected several tens of genes that are 


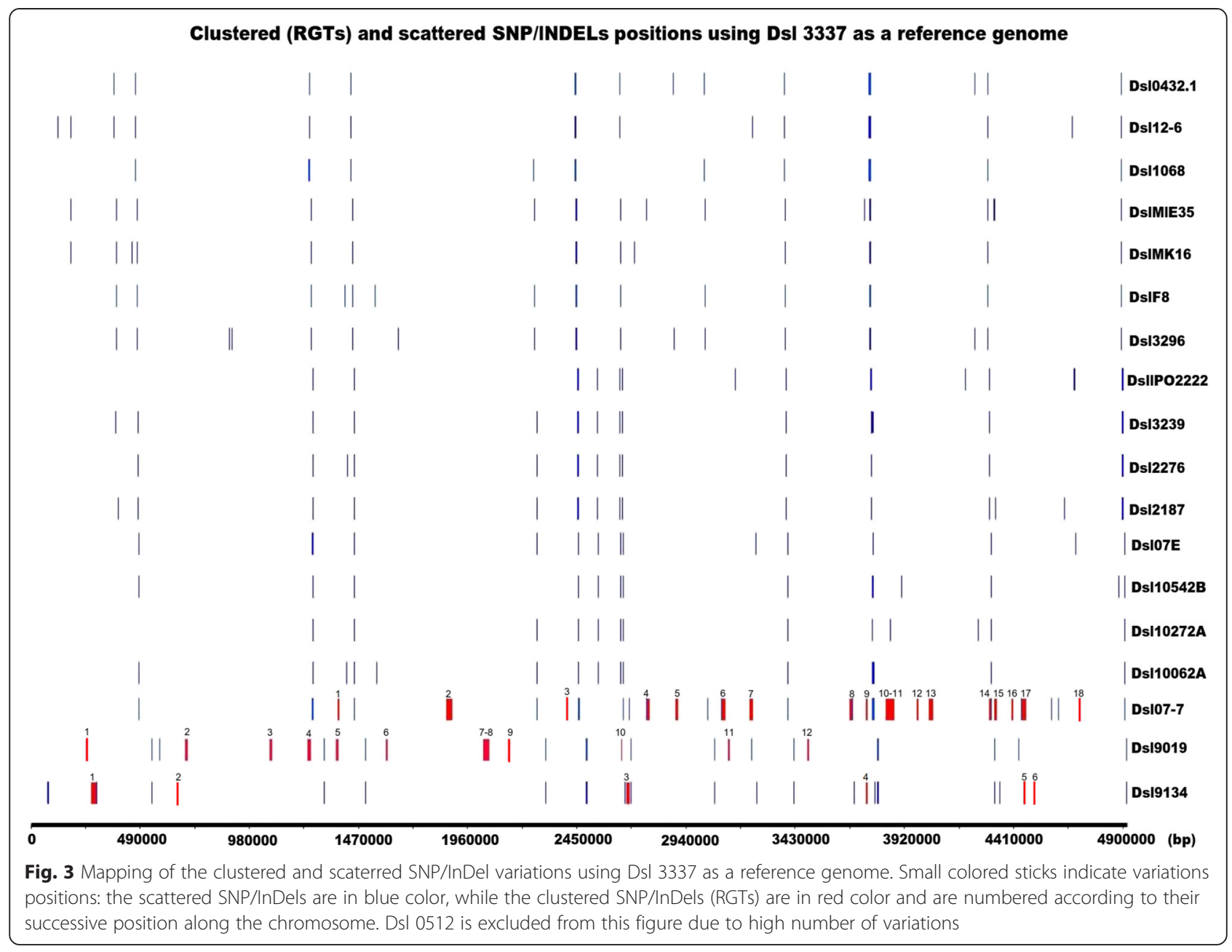

clustered in distinct regions, while only a few variations remain scattered. In Dsl 0512, variations exhibited a genome wide distribution. In the next part of the work, the three types of SNP/InDel distribution (scattered, clustered and wide genome distribution) have been analyzed in details.

\section{The mosaic genome of $\mathrm{D}$. solani 0512 might define a novel} D. solani sub-group

Dsl 0512 differed from the other D. solani by the high number and wide distribution of variations (Additional file 1: Table S3, Figs. 2 and 3), a unique phylogenetic position in MLSA (Fig. 1), and a high percentage (7.66 \%) of unmapped reads against Dsl 3337 genome (Additional file 1: Table S2). Unmapped reads were used for a de novo assembly which generated six contigs with a size ranging from $13248 \mathrm{bp}$ to $36630 \mathrm{bp}$. All these six contigs were absent from the other $D$. solani strains. Using MAUVE [24], these six sequences were positioned on the draft genome of Dsl 0512 that was constructed using the strain Dsl 3337 as a reference (Additional file 2: Figure S1). RAST annotation indicated that most of the genes belonging to these 6 contigs coded for phage elements and hypothetical or unknown proteins, with the exception of some genes coding for two putative $\mathrm{ABC}$ transporters, two putative virulence factors and one methyl-accepting chemotaxis protein, all being carried by the contig4. The similarity scores were too weak to assign a more precise function and phylogenetic origin to these putative genes/proteins.

Another characteristic of Dsl 0512 was a high number of genes (half of the genome) that exhibited variations. These genes were distributed along the genome without any clustering in specific regions. Constructed phylogenetic trees revealed that the analyzed genes exhibiting a nucleotide identity below 98 \% (compared to Dsl 3337 genes) did not belong to the core population gene cluster (Fig. 4, Additional file 3: Figure S2, Additional file 4: Figure S3, Additional file 5: Figure S4, Additional file 6: Figure S5). All these features supported the existence of a novel $D$. solani sub-group. The strain Dsl 0512 could be proposed as the eponym of the $D$. solani 0512 sub-group.

\section{Infra-species replacing HGT in DsI 07-7}

The 144 variant genes of Dsl 07-7 showed a non-uniform distribution along the chromosome, since most of them 


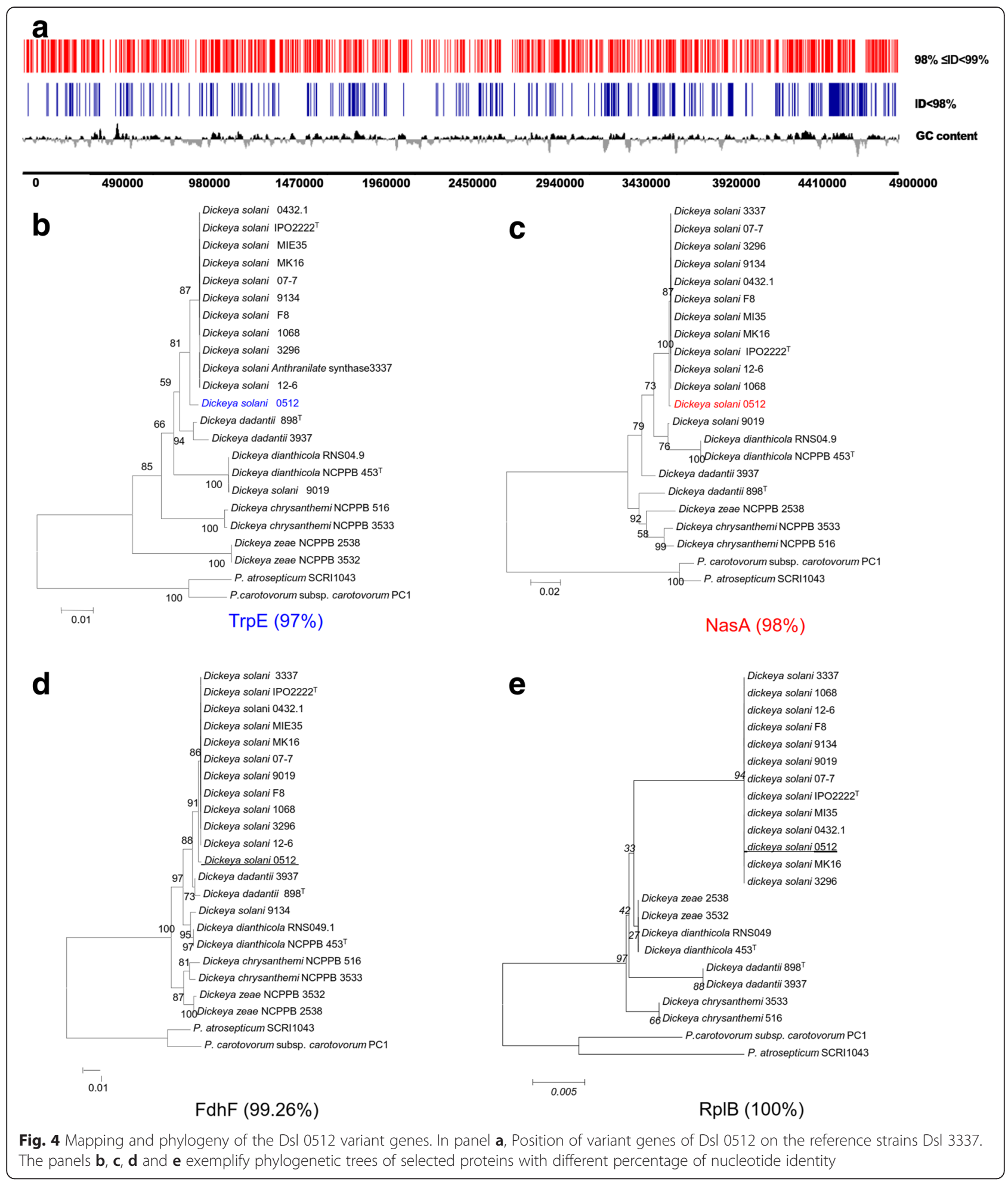

were clustered in 18 separate regions. One of these regions, which is presented in Fig. 5, contained four genes: oppB, oppF, an $\mathrm{ABC}$ transporter gene and $a m N$. These genes contained in total 47 variations leading to a decrease of their nucleotide identity as compared to the corresponding genes in the Dsl 3337 genome. Moreover, the phylogenetic analysis of the protein sequence coded by the $o p p B$ and $o p p F$ genes, which were 


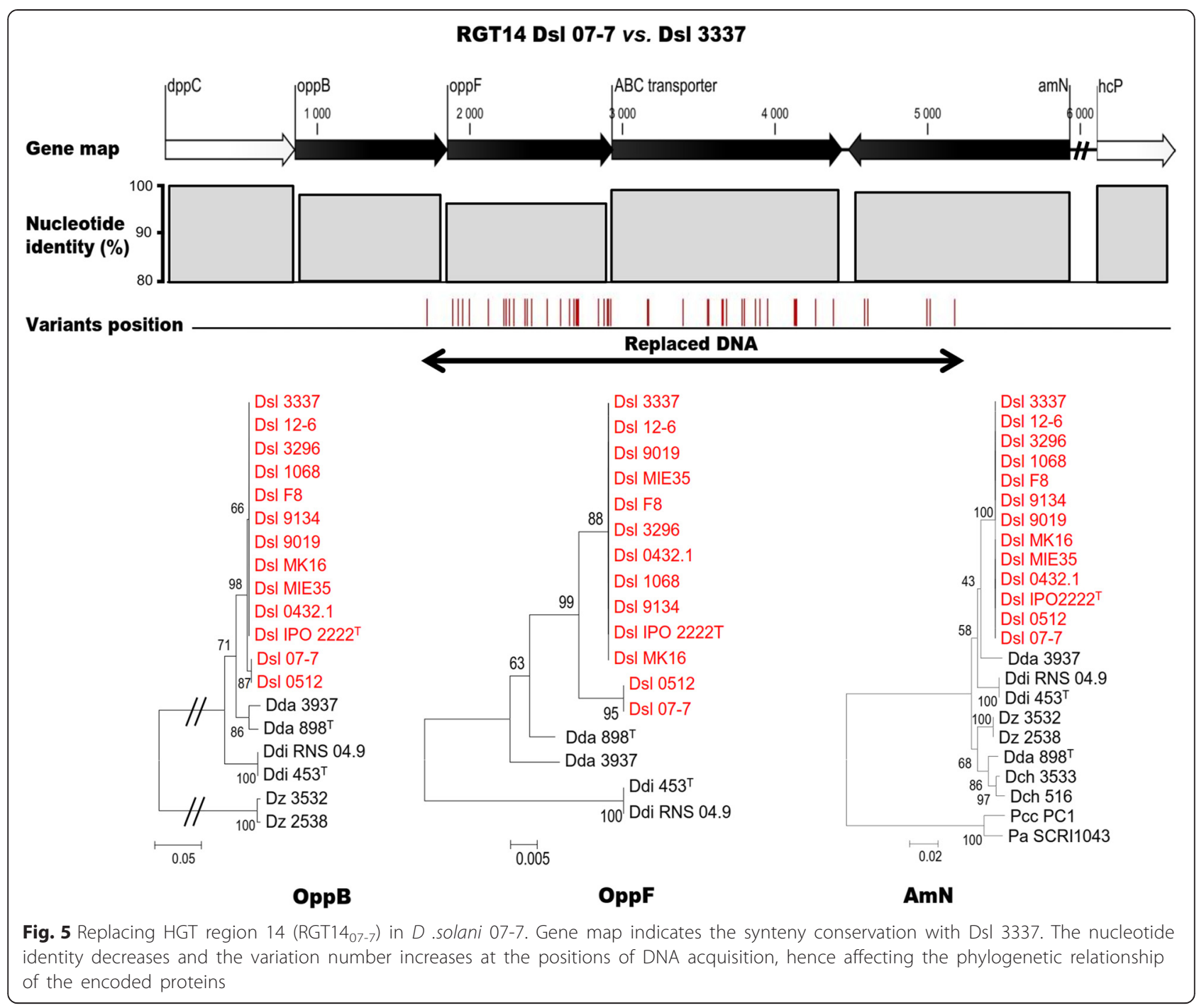

the most affected by variations, revealed a replacing HGT from a strain belonging to the D. solani 0512 subgroup. The 17 other regions exhibited a similar gene organization and a phylogenetic clustering with Dsl 0512 genes. Hence, all these 18 regions were called as RGT (replacing HGT) regions. They were numbered according to their successive position along the chromosome with the strain name in subscript position: $\mathrm{RGT}_{107-7}, \mathrm{RGT}_{207-7}, \mathrm{RGT}_{07-7} \ldots$ (Fig. 3). This analysis suggested that Dsl 07-7 acquired a dozen of gene fragments during massive replacing HGT(s) from strain(s) belonging to the Dsl 0512 sub-group. Hence, Dsl 07-7 exemplified the occurrence of an infra-specific gene exchange among the D. solani population, and also supported the possible co-existence of strains of the $D$. solani 0512 sub-group with those of the corepopulation.
Inter-species replacing HGT in D. solani strains 9134 and 9019

In Dsl 9134, 39 among the 56 genes with variations were clustered in 6 RGT regions, the other genes with variations being scattered along the chromosome. In Dsl 9019, 63 among the 73 genes with variations were clustered in 12 RGT regions. In both strains, the RGT regions were named according to the same nomenclature as in Dsl 07-7 (Fig. 3).

The RGT4 $4_{9134}$ illustrated the typical organization of these RGTs in Dsl 9134 (Fig. 6). RGT4 $4_{9134}$ (4860 bp) exhibited 229 positions of variations that were distributed in three genes: norF, norR, and fumA. These genes were related to the nitric oxide metabolism. Because of the high number of variations, the gene identity with $D$. solani strain 3337 decreased in RGT4 $4_{9134}$, especially in the norR gene that was located in the central part of 


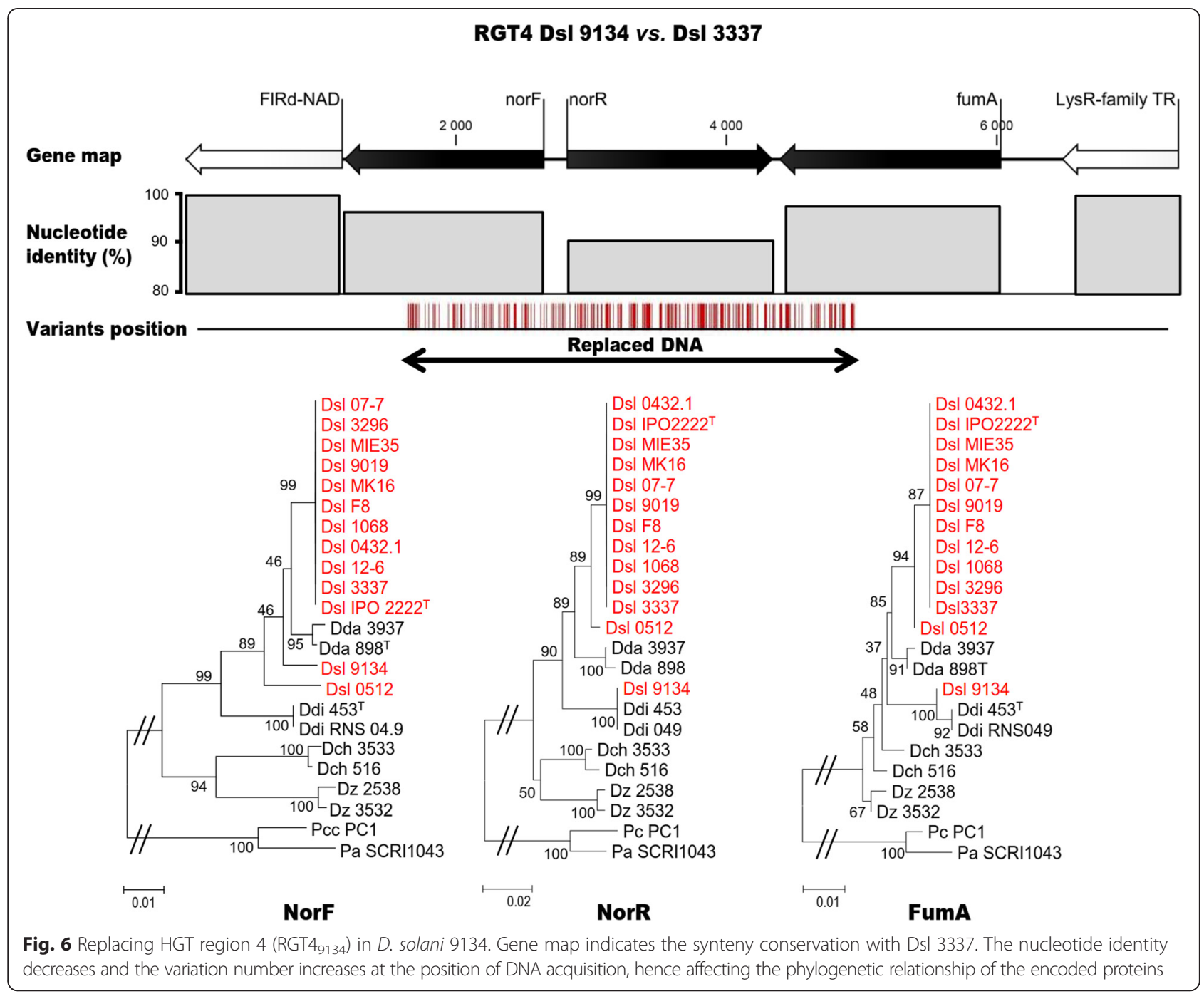

the RGT region. Protein phylogeny revealed that the three proteins encoded by the RGT4 $4_{9134}$ genes did not branch with their $D$. solani counterparts but were most closely related to those of $D$. dianthicola. The variation positions suggested that replacing HGT occurred in the middle of the genes norF and fumA, and hence generated proteins with an intermediate position between the $D$. solani and D. dianthicola proteins in the phylogenetic trees. A second example of inter-species replacing HGT is given with the RGT7 9019 (6248 bp) of Dsl 9019, which contained five genes dnaJ, dnaK, yaaH, a MFS transporter gene and $\operatorname{mog} A$ (Fig. 7). This example highlighted that replacing HGT might also affect genes such as dnaK and dnaJ which are used for MLSA and taxonomic identification [25]. In RGT7 9019,269 variants were detected. Discrepancies within DnaJ, DnaK and MogA phylogenies suggested the occurrence of a replacing HGT from D. dianthicola. In all the other RGTs of Dsl 9134 and 9019, a phylogeny approach (Additional file 5:
Figure S4, Additional file 6: Figure S5) also supported the occurrence of a replacing HGT using $D$. dianthicola population as the unique source.

\section{Plasmid acquisition in D. solani strain 9019 from Burkholderia}

In addition to replacing HGT, an additive HGT event that consisted in a plasmid acquisition occurred in Dsl 9019. The Dsl 9019 unmapped reads, which represented $1.9 \%$ of the total read number (Additional file 1: Table S2), allowed the generation of a single contig (43 $564 \mathrm{bp})$ by de novo assembly. This plasmid exhibited a complete identity (100 \%) with a plasmid of Bulkholderia ambifaria AMMD (CP000443.1). The stable replication of this plasmid in Dsl 9019 was verified in sub-cultures using plasmid-specific primers ( $\mathrm{pF} 1$ : cagcgaagagcaagac aa, pR1: tcatggaagcgatctcgg and pF2: ttaccggacgccga gctgtggcgt, pR2 :caggaagatgtcgttatcgcgagt). 


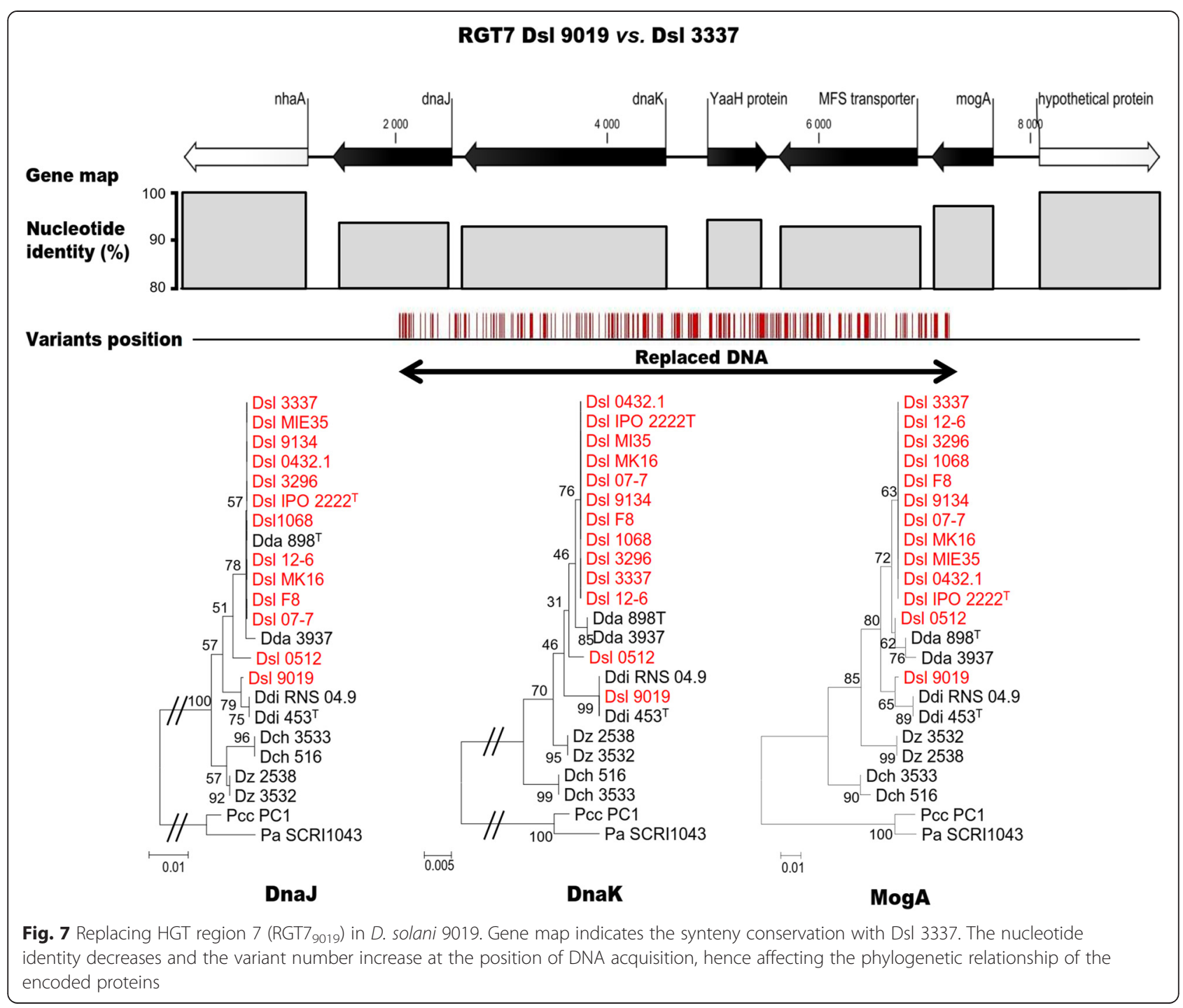

In D. solani 3296, variations in flagellar genes correlated motility and virulence decrease

All the non-synonymous variations of the core-population were listed in Additional file 1: Table S4. Remarkably, two unique non-synonymous variations that affected the $\mathrm{fliC}$ and fliN flagellar genes were present in Dsl 3296. The substitution $\mathrm{C}$ to $\mathrm{T}$ at the position 952985 lead to conversion of Ala207 to Thr in FliC, while deletion of the GTC codon starting at the position 966038 provoke the loss of the Val112 in FliN. The nucleotide variations were verified by Sanger sequencing. These two variations were unique among the sequenced $D$. solani strains, as well as the known Dickeya genomes (Additional file 7: Figure S6). These genes retained our attention as fli genes are required for aggressiveness in Dickeya and in other Enterobaceteriaceace [26-29]. We hypothesized that Dsl 3296 could be impaired in motility, hence also exhibited a reduced aggressiveness on potato host plants. We compared motility and virulence of all the 20 Dsl analyzed in this study (Fig. 8). All strains except Dsl 3296 were motile. Moreover, a weak aggressiveness of the strain 3296 was observed in virulence assay on potato tuber, hence correlating genomic variants in $f l i$ genes with motility and virulence deficiency. As a consequence, even if SNP/InDel variations are scarce, some of them may affect virulence functions in Dsl strains.

\section{Discussion}

This work provided new insights into the analysis of the emerging plant-pathogen $D$. solani. We combined Illumina and PacBio technologies to determine a high quality genome sequence of $D$. solani 3337 that we used as a reference to compare 19 other genome sequences generated by Illumina technology. While previous studies reported pairwise comparison between a single $D$. solani genome with that of other Dickeya and Pectobacterium species [13, 14], this work was also based on a population genomic approach. 


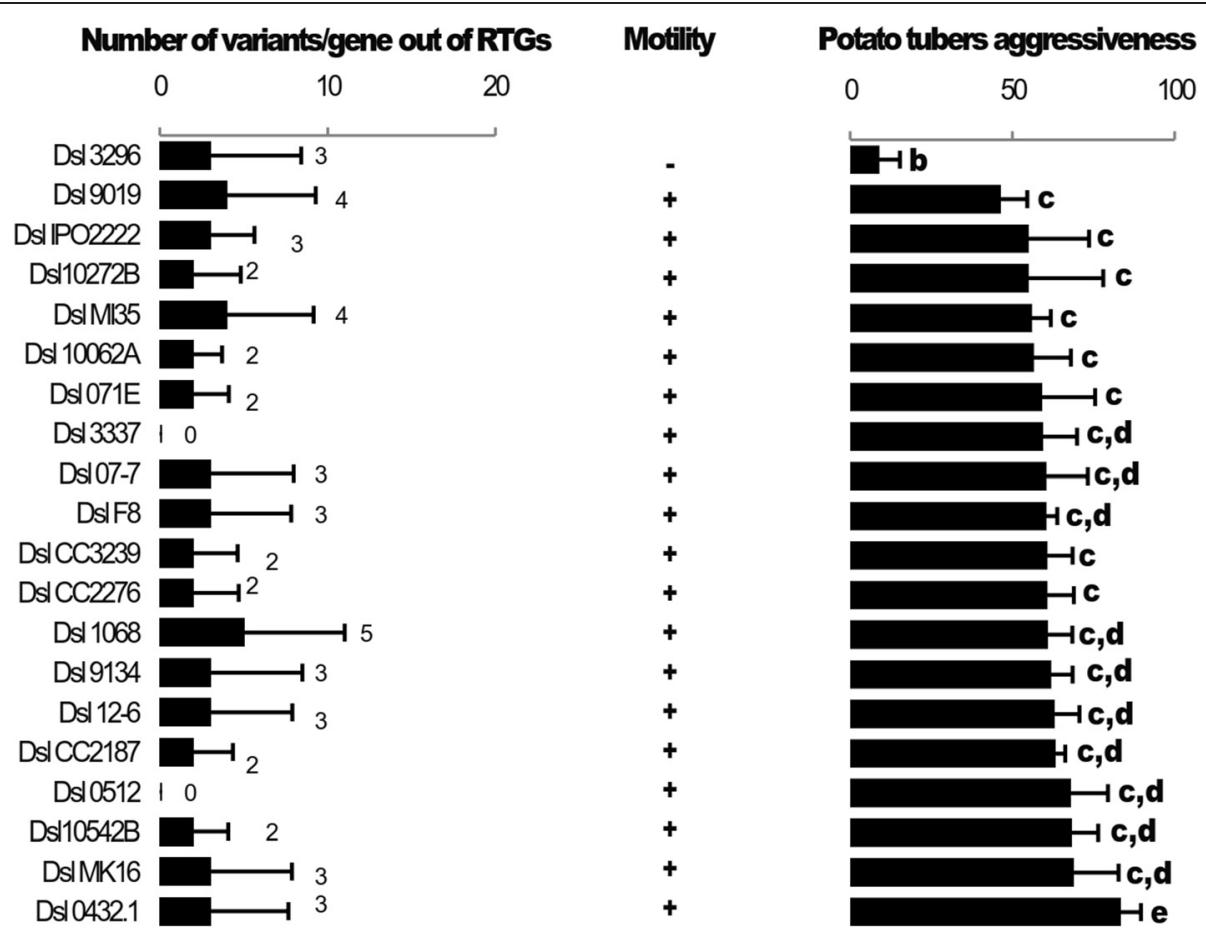

Fig. 8 Motility and aggressiveness assays performed on potato tubers. The average of variants per gene was calculated for each strain (the RGT regions of the strain Dsl 9019, 9134 and 07-7 were omitted for calculation). The signs + and - indicate that the strain is motile or not. The letters b, c, $d$ and e indicate statistical significance at $p<0.05$ (Kruskal-walis and Tukey tests) of the aggressiveness which was measured by infecting 30 potato tubers by each of the Dsl strains

This approach revealed the unexpected diversity of the $D$. solani genomes that resulted from a combination of scattered SNP/InDel variations as well as replacing and additive HGT events.

The majority of analyzed D. solani strains (16 among 20) that we called the core-population contained only 43 to 85 variants (SNPs and InDels). This result is in accordance with the high ANI values (>99.9 \%) that were calculated between each strain against the reference 3337. Other studies have pointed the high homogeneity within genetic equipment of $D$. solani population $[9,12,14,30]$. All these molecular analyses support the clonal hypothesis of the $D$. solani population. In spite of this high homogeneity, Dsl strains may exhibit some variability in aggressiveness on potato tubers [7]. A previous pairwise comparative study of two Dsl strains did not succeed in the identification of the genes and functions that could explain the different aggressiveness trait [7]. However, using a population comparative approach, we pointed out that genetic and functional variations in the motility trait could contribute to an aggressiveness decrease. This observation exemplifies the powerfulness of genomic diversity analyses on field isolates for the identification of genes that modulate aggressiveness.

Another important result was the characterization of a sub-group within the $D$. solani species, highlighting that
D. solani population structure was more complex than described previously. The prototypic strain of this subgroup was Dsl 0512 (RNS 05.1.2A) that has been isolated from potato plant showing blackleg and soft rot symptoms in France (in 2005). The existence of the 0512 sub-group was supported by ANI value, MLSA, genomic architecture (presence of specific regions) and SNP/InDel abundance and distribution. The Dsl 0512 genome appeared as a mosaic of genes with a phylogenetic position inferred to either the D. solani core population or the Dsl 0512 subgroup. Remarkably, genes that belong to the Dsl 0512 phylogenetic sub-cluster have also been discovered in the 18 RGTs (143 genes) of the strain Dsl 07-7 that was also isolated in France. The involvement of the 0512 subgroup as a gene resource in replacing HGT reinforced its importance in the generation of variability in $D$. solani isolates. The strains Dsl 0512 and 07-7 showed aggressiveness level similar to that of most of the studied $D$. solani strains, suggesting that the 0512 sub-group is not associated to any particular aggressiveness behavior, at least on potato tubers.

This study also highlighted that additive and replacing HGT occurred in inter-species exchanges. Additive HGT was observed in the strain 9019 which acquired a plasmid from B. ambifaria AMMD. B. ambifaria AMMD was isolated from the rhizosphere of healthy pea plants in 
Wisconsin (USA) in 1985 [31] and it has been reported as very effective in controlling phytopathogenic Pythium species [32]. Moreover we discovered replacing HGT events that recruited $D$. dianthicola genes in the two strains Dsl 9019 (63 genes distributed in 12 RGT regions) and 9134 (39 genes in 6 RGT regions) isolated from ornamental plants (respectively Muscari and Hyacynth). These exchange events between $D$. solani and $D$. dianthicola suggested that these two pathogens could coexist in the same ecological niche. In the case of Pectobacterium spp., multiple species isolations from the same infected plants have been reported [33, 34]. Importantly, the replacing HGT events did not correlate with an aggressiveness increase in Dsl 9019 and 9134 at least in potato tubers. However, replacing HGT generates major impact on phylogenetic inference by generating incongruities that could impair pathogen molecular diagnostics which are based on housekeeping genes. Importantly, it has been reported that succeeded HGT events between distantly related bacteria mostly implicate housekeeping genes that are also the most conserved between different species [35, 36]. Our work revealed that in the Dsl 9019 strain, the dnaJ and dnaK genes, which are usually used in phylogenetic classifications [25, 37], have been recruited from $D$. dianthicola. Since the Dickeya pathogens are genetically very close (ANI $\geq 93 \%$ ), replacing HGT could be predicted to interfere recurrently with taxonomical diagnostics, hence provoking assignation errors. The impact of HGT on taxonomy has been discussed in different Enterobacteriaceae $[38,39]$. An immediate applied recommendation from our work is that even though $D$. solani is mainly described as a homogeneous population, the existence of HGT events should encourage the use of multiple taxonomical markers.

\section{Conclusions}

As a conclusion, this work revealed that $D$. solani genomic variability may be caused by SNPs/InDels as well as replacing and additive HGT events, including plasmid acquisition. From this work, the question arises about the dynamics of the $D$. solani diversity in the course of its emergence and spreading in crop cultures. This might be further investigated by a larger scale sampling and genomic analysis.

\section{Methods}

\section{Bacterial strains and growth conditions}

$D$. solani strains were collected from different geographical locations and dates of isolation and also from different hosts or environments (Additional file 1: Table S1). All the strains were routinely cultured in TY medium (tryptone $5 \mathrm{~g} / \mathrm{L}$, yeast extract $3 \mathrm{~g} / \mathrm{L}$ and agar $1.5 \%)$ at $28{ }^{\circ} \mathrm{C}$.

\section{DNA extraction and sequencing}

Genomic DNA from each strain was extracted from overnight culture using a phenol-chloroform purification method followed by an ethanol precipitation as described by [40] Wilson. Quantity and quality control of the DNA was completed using a NanoDrop (ND 1000) device and agarose gel electrophoresis at $1.0 \%$.

Paired-end libraries with an insert size of 270 to 390 bp were constructed for each strain, and DNA sequencing was performed by Illumina HiSeq 2000 v3 technology. Sequencing of the library was carried out using $2 \times 100$ or $2 \times 150$ bp paired-end read module. Illumina sequencing was performed at the CNRS IMAGIF platform (Gif-sur-Yvette).

The genomic DNA of Dsl 3337 was subjected to PacBio RSII sequencing technology (Pacific Biosciences, CA, USA) using library targeted at $10 \mathrm{kbp}$ in insert size. Prior to assembly, short reads that are less than 500 bp were filtered off and minimum polymerase read quality used for mapping of subreads from a single zero-mode waveguides (ZMW) was set at 0.75 . The 112228 filtered reads (N50 value was 13159 bp and total bp number was 814445 948) were assembled using RS_HGAP_Assembly (version 3.0), which is an analysis pipeline module from Pacific Biosciences SMRT portal incorporating Celera Assembler, BLASR mapper and Quiver consensus caller algorithm. The cut-off length of seeding reads was set at $3606 \mathrm{bp}$ in order to serve as a reference for the recruitment of shorter reads for preassembly. The resulted consensus accuracy based on multiple sequence alignment of the subreads was at $99.99 \%$.

Assembly, variants calling and genome sequence analysis Assembly of the sequences was performed using the CLC Genomics Workbench v7.0.0 software (CLC Inc, Aarhus, Denmark). After quality (quality score threshold 0.05 ) and length (above 40 nucleotides) trimming of the sequences, contigs were generated by de novo assembly (CLC parameters: automatic determination of the word and bubble sizes with no scaffolding) for each strain.

Paired end reads for each strain were mapped against the reference sequence of the strain $D$. solani 3337 at mild stringency threshold ( 0.8 of identity on 0.5 of read length) using CLC Genomics Workbench version 7.0.0 software. The unmapped reads for each strain were collected. The mappings were used for detection of variations (SNPs and InDels) using basic variant calling tool from CLC genomic workbench version 7.0.0. Draft genome sequences composed of the contigs of each strain were used to search and analyze the variations detected. Variations with an occurrence below $99 \%$ in the mapping step were discarded from the study.

The nucleotide identity (ANI) values were calculated as previously proposed [41] using the ANI calculator 
from the Kostas lab with default settings (http://enveomics.ce.gatech.edu/ani/). Phylogenetic and molecular evolutionary analyses were conducted using MEGA, version 6 [23]. An MLSA (Multi-locus sequence analysis) was performed using eleven housekeeping genes $(r p o D$, gyrB, recA, rpoS, dnaX, dnaA, gapA, fusA, rplB, purA, gyr $A$ ) retrieved from the twenty $D$. solani strains in order to confirm their phylogenetic position within known pectinolytic Dickeya and Pectobacterium strains.

\section{Nucleotide sequence accession number}

Draft genome sequences of Dickeya solani strains 9109, 0512, 9134, 07-7 have been deposited at DDBJ/EMBL/ GenBank under the following accession numbers: (JWLS00000000) D. solani 9019, (JWMJ00000000) D. solani 0512, (JWLT00000000) D. solani 9134, (JWLR 00000000) D. solani 07-7. The versions described in this paper are versions (JWLS01000000) D. solani 9019, (JWMJ01000000) D. solani 0512, (JWLT01000000) D. solani 9134, (JWLR01000000) D. solani 07-7. Genomes of other Dickeya and Pectobacterium species were collected from public database (Table S4).

\section{Aggressiveness and motility assays}

Motility assays were conducted on semi-solid SM medium (beef extract at $3 \mathrm{~g} / \mathrm{L}$, peptone at $5 \mathrm{~g} / \mathrm{L}$, and $25 \mathrm{ml} / \mathrm{L}$ of $20 \%$ glucose) with $0.5 \%$ of agar. Two $\mu \mathrm{L}$ of an overnight bacterial suspension of each strain were used to inoculate agar plates which were incubated $16 \mathrm{~h}$ at $28{ }^{\circ} \mathrm{C}$. The experiment was performed twice with 2 replicates each time.

Assessment of the aggressiveness of the strains was performed on potato tubers (cv. Binjte). To this end, $10^{6} \mathrm{CFU}$ were used to infect 10 potato tubers for each strain. After $24 \mathrm{~h}$ of incubation at $25^{\circ} \mathrm{C}$, five aggressiveness categories were considered and attributed to tuber samples to assess the virulence of the strains. The experiments were performed three times, hence 600 tubers were infected and analyzed. The results were represented as normalized values.

Virulence assays were statistically analyzed to infer the aggressiveness variability within strains on potato tubers. Heterogeneity of strains was assessed using a KruskalWalis test with $\mathrm{p}<0.05$. Statistical significance of the pairwise comparisons between strains was calculated using a post hoc Tukey test with $\mathrm{p}<0.05$.

\section{Availability of supporting data}

The alignments and phylogenetical tree for MLSA are available through the Dryad data repository doi: 10.5061/dryad.h26hs.

\section{Additional files}

\begin{abstract}
Additional file 1: Tables S1-S5. Table S1. Dickeya solani strains in this study. Table S2. Sequencing data and mappings on the Dsl 3337 genome. Table S3 Variants distribution on the strains vs. Dsl 3337. Table S4. Non-synonymous variants: this table shows the unique and the shared variants within homoge:nous D. solani strains (MK16, MIE35, 0432.1, 12-6, F8, 1068, 3296, 07E, 10062A, 10272B, 10542B, 2187, 2276, 3239, IPO2222 ${ }^{\top}$ ). Table S5. Other genomes used in this study (DOC $130 \mathrm{~kb}$ )
\end{abstract}

Additional file 2: Figure S1. Synteny between the strain D. solani 3337 and the draft genome D. solani 0512. The alignment was performed using MAUVE software, underlining a high conservation of the synteny. The numbers indicate the positions of the strain-specific genomic regions generated by de novo assembly of the unmapped reads. (TIFF $956 \mathrm{~kb}$ )

Additional file 3: Figure S2. Protein-based phylogenetic trees revealing Dsl 0512 as a member of in distinct sub-cluster within the $D$. solani species. (TIFF $2843 \mathrm{~kb}$ )

Additional file 4: Figure S3. Protein-based phylogenetic trees of different RGTs in Dsl 07-7. The genes were retrieved from RGT1, RGT2, RGT3, RGT6, RGT10 and RGT12 of Dsl 07-7. The phylogenetic positions indicate replacing HGT events from the D. solani 0512 sub-group. (TIFF $2881 \mathrm{~kb}$ )

Additional file 5: Figure S4. Protein-based phylogenetic trees of different RGTs in Dsl 9134. The genes were retrieved from RGT1, RGT3, RGT5 and RGT6 of Dsl 9134. The phylogenetic positions highlight replacing HGT events from the $D$. dadantii species. (TIFF $2117 \mathrm{~kb}$ )

Additional file 6: Figure S5. Protein-based phylogenetic trees of different RGTs in Dsl 9019. The genes were retrieved from RGT1, RGT2, RGT3, RGT4, RGT5 and RGT10 of Ds19019. The phylogenetic positions highlight replacing HGT events from the D. dadantii species. (TIFF 2989 kb)

Additional file 7: Figure S6. Local alignment of FliC and FliN proteins. The variations at the positions 207 in FliC and 112 in FliN are indicated in red color, other variations are in blue color. Amino acid position is numbered according to the D. solani 3337 sequence of FliC and FliN. We used the draft and complete genomes of the $19 \mathrm{D}$. solani sequenced in this study, those of D. solani strains GBCC2040 and MK10, 15 D. dianthicola including the strains MIE32, MIE33, MIE34, CFBP1888, CFBP2015, CFBP2982, RNS04.9, RNS10.20.2A, RNS11.47.1A, DW04.9 K, DS05.3.3, GBBC2039, IPO980, NBPPB3534 and NCPPB453, D. dadantii strains 3937, NCPPB898 and NCPPB3537, D. chrysanthemi strains NCPPB3533 and NCPPB516, and D. zeae strains Ech1591 and NCPPB2538. (TIFF $1283 \mathrm{~kb}$ )

\section{Abbreviations}

ANI: Average nucleotide identity; CDS: Coding DNA sequence; Dsl: Dickeya solani; HGT: Horizontal gene transfer; InDel: Insertion deletion; MLSA: Multi-locus sequence analysis; NCBI: National center for biotechnology information; RGT: Replacing HGT; SNP: Single nucleotide polymorphism.

\section{Competing interests}

The authors declare that they have no competing interests.

\section{Authors' contributions}

SK performed comparative genomics and phylogenetic analyses, PB performed virulence assays and DNA extractions, JP and FVG analyzed genomic data, TMC and KGC carried out PacBio sequencing, VH and FVG provided strains, DF coordinated the project, and all the authors, SK, PB, JP, FVG, TMC, KGC, VH, DF and MM contribute in manuscript writing. All the authors read and approved the final manuscript.

\section{Acknowledgements}

We thank Robert Dees (Wageningen UR/Applied Plant Research) for the gift of the ornamental strains D. solani PPO9019 and PPO9134, Minna Pirhonen (Department of Applied Biology, University of Helsinki) for providing PPL0433 (=F8), Leah Tsror (Gilat Research Center, Agricultural Research Organisation) for providing GRC77 (EU3296) and Yves Dessaux (I2BC, CNRS) for his help in the manuscript editing. This work was supported by a cooperative project between France and Morocco (PRAD 14-02, Campus France $n^{\circ} 30229$ ZK), the University Paris-Saclay (Co-tutelle funding), the excellence grant ( $\left.n^{\circ} \mathrm{H} 011 / 007\right)$ awarded by the Ministry of Higher education of Morocco, a collaborative project between 
CNRS (Gif sur Yvette) and FN3PT-RD3PT (Paris), the High Impact Research Grant (UM.C/625/1/HIR/MOHE/CHAN/14/01, Grant number A-000001-50001 to KGC) and the French-Malaysian exchange program awarded by French Embassy of Malaysia.

\section{Author details}

${ }^{1}$ Institute for Integrative Biology of the Cell (I2BC), CNRS CEA Univ. Paris-Sud, Université Paris-Saclay, Saclay Plant Sciences, Avenue de la Terrasse, 91198 Gif-sur-Yvette cedex, France. ${ }^{2}$ Université Moulay Ismaïl, Faculté des Sciences, Département de Biologie, Meknès, Morocco. ${ }^{3}$ UPMC Univ Paris 06, UMR 7618, IEES Paris (Institute of Ecology and Environmental Sciences), 7 Quai Saint bernard, 75005 Paris, France. ${ }^{4}$ Division of Genetics and Molecular Biology, Institute of Biological Sciences, Faculty of Science, University of Malaya, 50603 Kuala Lumpur, Malaysia. ${ }^{5}$ Fédération Nationale des Producteurs de Plants de Pomme de Terre-Recherche développement Promotion du Plant de Pomme de Terre (FN3PT-RD3PT), 75008 Paris, France. ${ }^{6}$ UMR 1349 IGEPP

INRA - Agrocampus Ouest Rennes, 35653 LeRheu, France. ${ }^{7}$ INRA, UMR 1392, IEES Paris (Institute of Ecology and Environmental Sciences), 7 Quai Saint Bernard, 75005 Paris, France.

Received: 19 June 2015 Accepted: 3 October 2015 Published online: 14 October 2015

\section{References}

1. Samson R. Transfer of Pectobacterium chrysanthemi (Burkholder et al. 1953) Brenner et al. 1973 and Brenneria paradisiaca to the genus Dickeya gen. nov. as Dickeya chrysanthemi comb. nov. and Dickeya paradisiaca comb. nov. and delineation of four novel species, Dickeya dadantii sp. nov., Dickeya dianthicola sp. nov., Dickeya dieffenbachiae sp. nov. and Dickeya zeae sp. nov. Int J Syst Evol Microbiol. 2005;55:1415-27.

2. Gardan L. Elevation of three subspecies of Pectobacterium carotovorum to species level: Pectobacterium atrosepticum sp. nov., Pectobacterium betavasculorum sp. nov. and Pectobacterium wasabiae sp. nov. Int I Syst Evol Microbiol. 2003;53:381-91.

3. Collmer A, Keen NT. The role of pectic enzymes in plant pathogenesis. Annu Rev Phytopathol. 1986;24:383-409.

4. Toth IK, van der Wolf JM, Saddler G, Lojkowska E, Hélias V, Pirhonen M, et al. Dickeya species: an emerging problem for potato production in Europe: Dickeya spp. on potato in Europe. Plant Pathol. 2011;60:385-99.

5. Van der Wolf JM, Nijhuis EH, Kowalewska MJ, Saddler GS, Parkinson N, Elphinstone JG, et al. Dickeya solani sp. nov., a pectinolytic plant-pathogenic bacterium isolated from potato (Solanum tuberosum). Int J Syst Evol Microbiol. 2014;64:768-74.

6. Czajkowski R, de Boer WJ, van der Zouwen PS, Kastelein P, Jafra S, de Haan EG, et al. Virulence of "Dickeya solani" and Dickeya dianthicola biovar-1 and -7 strains on potato (Solanum tuberosum): Comparison of Dickeya spp. on potato. Plant Pathol. 2013;62:597-610.

7. Potrykus M, Golanowska M, Hugouvieux-Cotte-Pattat N, Lojkowska E. Regulators Involved in Dickeya solani Virulence, Genetic Conservation, and Functional Variability. Mol Plant Microbe Interact. 2014;27:700-11.

8. Czajkowski R, de Boer WJ, Velvis H, van der Wolf JM. Systemic colonization of potato plants by a soilborne, green fluorescent protein-tagged strain of Dickeya sp. biovar 3. Phytopathology. 2010;100:134-42.

9. Laurila J, Ahola V, Lehtinen A, Joutsjoki T, Hannukkala A, Rahkonen A, et al. Characterization of Dickeya strains isolated from potato and river water samples in Finland. Eur J Plant Pathol. 2008;122:213-25.

10. Sławiak M, van Beckhoven JRCM, Speksnijder AGCL, Czajkowski R, Grabe G, van der Wolf JM. Biochemical and genetical analysis reveal a new clade of biovar 3 Dickeya spp. strains isolated from potato in Europe. Eur J Plant Pathol. 2009;125:245-61.

11. Parkinson N, Stead D, Bew J, Heeney J, Tsror Lahkim L, Elphinstone J. Dickeya species relatedness and clade structure determined by comparison of recA sequences. Int J Syst Evol Microbiol. 2009;59(Pt 10):2388-93.

12. Van Vaerenbergh J, Baeyen S, De Vos P, Maes M. Sequence Diversity in the Dickeya fliC Gene: Phylogeny of the Dickeya Genus and TaqMan ${ }^{\circledR}$ PCR for "D. solani", New Biovar 3 Variant on Potato in Europe. PLoS ONE. 2012; $7:$ e35738.

13. Garlant $L$, Koskinen $P$, Rouhiainen $L$, Laine P, Paulin L, Auvinen P, et al. Genome sequence of Dickeya solani, a new soft rot pathogen of potato, suggests its emergence may be related to a novel combination of nonribosomal peptide/polyketide synthetase clusters. Diversity. 2013;5:824-42.

14. Pédron J, Mondy S, Raoul des Essarts Y, Van Gijsegem F, Faure D. Genomic and metabolic comparison with Dickeya dadantii 3937 reveals the emerging Dickeya solani potato pathogen to display distinctive metabolic activities and T5SS/T6SS-related toxin repertoire. BMC Genomics. 2014;15:283.

15. Smith MW, Feng D-F, Doolittle RF. Evolution by acquisition: the case for horizontal gene transfers. Trends Biochem Sci. 1992;17:489-93.

16. Smith JM, Smith NH, O'Rourke M, Spratt BG. How clonal are bacteria? Proc Natl Acad Sci U S A. 1993;90:4384-8.

17. Ochman H, Lawrence JG, Groisman EA. Lateral gene transfer and the nature of bacterial innovation. Nature. 2000;405:299-304.

18. Koonin EV, Makarova KS, Aravind L. Horizontal gene transfer in prokaryotes: quantification and classification. Annu Rev Microbiol. 2001;55:709-42.

19. Frost LS, Leplae R, Summers AO, Toussaint A. Mobile genetic elements: the agents of open source evolution. Nat Rev Microbiol. 2005;3:722-32.

20. Juhas M, van der Meer JR, Gaillard M, Harding RM, Hood DW, Crook DW. Genomic islands: tools of bacterial horizontal gene transfer and evolution. FEMS Microbiol Rev. 2009:33:376-93.

21. Choi SC, Rasmussen MD, Hubisz MJ, Gronau I, Stanhope MJ, Siepel A. Replacing and additive horizontal gene transfer in Streptococcus. Mol Biol Evol. 2012;29:3309-20.

22. Khayi S, Mondy S, Beury-Cirou A, Moumni M, Helias V, Faure D. Genome sequence of the emerging plant pathogen Dickeya solani strain RNS 08.23.3.1A. Genome Announc. 2014;2:e01270-13.

23. Tamura K, Stecher G, Peterson D, Filipski A, Kumar S. MEGA6: Molecular Evolutionary Genetics Analysis Version 6.0. Mol Biol Evol. 2013;30:2725-9.

24. Darling ACE. Mauve: multiple alignment of conserved genomic sequence with rearrangements. Genome Res. 2004;14:1394-403.

25. Colston SM, Fullmer MS, Beka L, Lamy B, Gogarten JP, Graf J. Bioinformatic genome comparisons for taxonomic and phylogenetic assignments using Aeromonas as a test case. mBio. 2014;5:e02136-14.

26. Rogers TJ, Paton JC, Wang H, Talbot UM, Paton AW. Reduced virulence of an fliC mutant of Shiga-toxigenic Escherichia coli O113:H21. Infect Immun. 2006;74:1962-6.

27. Jahn CE, Willis DK, Charkowski AO. The flagellar sigma factor FliA is required for Dickeya dadantii virulence. Mol Plant Microbe Interact. 2008;21:1431-42.

28. He $Y, X u T$, Fossheim LE, Zhang X-H. FliC, a flagellin protein, is essential for the growth and virulence of fish pathogen Edwardsiella tarda. PLOS ONE. 2012;7:e45070.

29. Dong T, Schellhorn HE. Role of RpoS in virulence of pathogens. Infect Immun. 2010;78:887-97.

30. Pritchard L, Humphris S, Saddler GS, Parkinson NM, Bertrand V, Elphinstone $J G$, et al. Detection of phytopathogens of the genus Dickeya using a PCR primer prediction pipeline for draft bacterial genome sequences: Dickeya diagnostics from draft bacterial genome sequences. Plant Pathol. 2013;62:587-96.

31. Winsor GL, Khaira B, Van Rossum T, Lo R, Whiteside MD, Brinkman FSL. The Burkholderia Genome Database: facilitating flexible queries and comparative analyses. Bioinforma Oxf Engl. 2008;24:2803-4.

32. Coenye T, Mahenthiralingam E, Henry D, LiPuma JJ, Laevens S, Gillis M, et al. Burkholderia ambifaria sp. nov., a novel member of the Burkholderia cepacia complex including biocontrol and cystic fibrosis-related isolates. Int J Syst Evol Microbiol. 2001;51:1481-90.

33. Ma B, Hibbing ME, Kim H-S, Reedy RM, Yedidia I, Breuer J, et al. Host range and molecular phylogenies of the soft Rot enterobacterial genera Pectobacterium and Dickeya. Phytopathology. 2007;97:1150-63.

34. Glasner JD, Marquez-Villavicencio M, Kim H-S, Jahn CE, Ma B, Biehl BS, et al. Niche-specificity and the variable fraction of the Pectobacterium pan-genome. Mol Plant-Microbe Interact. 2008;21:1549-60.

35. Jain R, Rivera MC, Lake JA. Horizontal gene transfer among genomes: The complexity hypothesis. Proc Natl Acad Sci U S A. 1999;96:3801-6.

36. Rivera MC, Jain R, Moore JE, Lake JA. Genomic evidence for two functionally distinct gene classes. Proc Natl Acad Sci U S A. 1998;95:6239-44.

37. Alexandre A, Laranjo M, Young JPW, Oliveira S. dnaJ is a useful phylogenetic marker for alphaproteobacteria. Int J Syst Evol Microbiol. 2008;58:2839-49.

38. Philippe H, Douady CJ. Horizontal gene transfer and phylogenetics. Curr Opin Microbiol. 2003;6:498-505. 
39. Shapiro BJ. Signatures of natural selection and ecological differentiation in microbial genomes. In: Landry CR, Aubin-Horth N, editors. Ecological genomics. Volume 781. Dordrecht: Springer Netherlands; 2014. p. 339-59.

40. Wilson K. Preparation of genomic DNA from bacteria. Curr Protoc Mol Biol. 1987; Chapter 2:2-4.

41. Goris J, Konstantinidis KT, Klappenbach JA, Coenye T, Vandamme P, Tiedje JM. DNA-DNA hybridization values and their relationship to whole-genome sequence similarities. Int J Syst Evol Microbiol. 2007;57:81-91.

Submit your next manuscript to BioMed Central and take full advantage of:

- Convenient online submission

- Thorough peer review

- No space constraints or color figure charges

- Immediate publication on acceptance

- Inclusion in PubMed, CAS, Scopus and Google Scholar

- Research which is freely available for redistribution 